\title{
Alter
}

Revue de phénoménologie

$28 \mid 2020$

La religion

\section{Du sacré au saint : ereignis et liturgie}

Sr Marie-Aimée Manchon

\section{(2) OpenEdition}

\section{Journals}

Édition électronique

URL : https://journals.openedition.org/alter/2056

DOI : 10.4000/alter.2056

ISSN : 2558-7927

\section{Éditeur :}

Association ALTER, Archives Husserl (CNRS-UMR 8547)

\section{Édition imprimée}

Date de publication : 1 novembre 2020

Pagination : 191-204

ISBN : 978-2-9550449-6-4

ISSN : 1249-8947

\section{Référence électronique}

Sr Marie-Aimée Manchon, «Du sacré au saint : ereignis et liturgie», Alter [En ligne], 28| 2020, mis en ligne le 22 décembre 2020, consulté le 15 octobre 2021. URL : http://journals.openedition.org/alter/ 2056 ; DOI : https://doi.org/10.4000/alter.2056

Ce document a été généré automatiquement le 15 octobre 2021

Revue Alter 


\title{
Du sacré au saint : ereignis et liturgie
}

\author{
Sr Marie-Aimée Manchon
}

1 Si le $\mathrm{XX}^{\mathrm{e}}$ siècle a entériné la «mort de Dieu » clamée haut et fort depuis le siècle précédent, il n'en a pas moins réinvesti aussi, et comme en contrepoint, de manière oblique ou frontalement, la dimension du sacré. Cela peut étonner, et, à vrai dire, cela étonne, ce qui signifie qu'il y a ici matière à philosopher. C'est ainsi qu'avec Rudolph Otto et Mircea Eliade, l'anthropologie s'est mise à étudier savamment les distinctions entre profane et sacré mises en place par "l'homo religiosus $\|^{1}$; la sociologie des religions fera son apparition sur cette lancée. En théologie, le Mouvement liturgique interroge lui aussi la dimension de sacré propre au culte chrétien : Odon Casel ose un parallèle entre liturgie chrétienne et religions antiques à mystères, repris et critiqué par Louis Bouyer qui souligne de son côté le lien entre sacré et sacrifice comme intrinsèque au mystère du culte lui-même ${ }^{2}$. Bouyer rappelle en ce sens l'inanité du "profane » dans l'expérience croyante : «le profane, à vrai dire, n'est jamais que du sacré profané. A l'origine de l'expérience humaine, en effet, c'est toute la réalité qui s'offre à elle qui constitue le domaine du sacré. Le sacré, encore une fois, c'est précisément l'appréhension originelle du monde par l'homme, sous l'aspect d'une totalité que l'homme saisit d'emblée comme qualitativement unique $»^{3}$. Nostalgique de cette unicité, la littérature de son côté n'est pas en reste, qui, après une reprise récurrente de thèmes chrétiens ${ }^{4}$, se délecte de revisiter la mythologie grecque. Que l'on songe seulement, rien que pour la figure d'Ulysse, à Giraudoux (Elpénor, 1919), Joyce (Ulysse, 1922), Giono (Naissance de l'Odyssée, 1938)... Au même moment en philosophie, Heidegger vit son «tournant » (Kehre) qui, tout déchristianisé qu'il se targue d'être, renoue avec une forme de sacré omniprésent à travers sa partition du monde en quatre dimensions (Geviert ou Quadriparti) inspirée de la poésie d'Hölderlin. Le monde pensé comme athée ne semble pas pouvoir se passer de sacré, et ce, dans toutes les formes de ce que l'on appelle les Humanités. Heidegger sera pour nous ici le prototype de cette embardée du sacré au moment même où est massivement refusée au réel toute transcendance, à Dieu toute consistance, et donc à la religion toute clairvoyance. Quand la foi est mise à mal, restent encore des croyances plus ou moins bien cernées. Mais ce sacré n'en appelle-t-il pas encore et toujours au saint qu'il dénie ? Telle est, sans doute, la leçon que, selon Jean-Yves Lacoste, la liturgie donne au Dasein, et plus 
particulièrement, selon nous, au concept qui focalise l'acception heideggérienne du sacré : l'Ereignis.

\section{La sacrée clairière de Heidegger}

2 Contre "la Machination » (Machenschaft) ou « la technique » (Gestell) qui génèrent la réification de l'homme, le sacré seul permettrait, selon Heidegger, de rendre vivable, habitable, le monde où nous sommes jetés. Il ne s'agit pas de renouer avec quelque religion que ce soit - toutes ont prouvé leur inanité pour le philosophe allemand. Mais il semble que ce lien qui est l'apanage de la religion (religare) si l'on en croit son étymologie, certes controversée ${ }^{1}$, nous est constitutif au moins sous la coordonnée du sacré. Car l'homme qui «mondanéise» (welten) ce qui l'entoure ne le fait pas sans la "mesure du ciel», que celui-ci soit habité par le Dieu absolu ou peuplé de divin impersonnel. "L'habitation de l'homme repose dans cette mesure aménageante qui regarde vers le haut, dans cette mesure de la Dimension où le ciel, aussi bien que la terre, a sa place " ${ }^{2}$. Alors peut avoir lieu (trouver son là) l'Ereignis, cet «événement » de l'advenue de l'Être enfin reconnu dans sa clarté (Lichtung) qui pourtant nous en préserve le secret (Geheimnis). Cet ajointement ou appropriement entre l'homme et l'être où la donation enfin accomplie peut se faire eschatologie de l'Être, autre commencement, saut dans l'originaire, hors de toute métaphysique classique désormais périmée, Heidegger en a fait un livre jamais publié de son vivant mais qu'il déclare ouvertement être le point focal de sa pensée depuis les années 1930. Beiträge zur Philosophie, vom Ereignis, écrits entre 1936 et 1938 sont désormais traduits aux éditions Gallimard par François Fédier en Apports à la philosophie, De l'avenance (1993).

Le néologisme avenance, certes, peut décontenancer, voire agacer, et beaucoup l'ont critiqué, mais il a au moins le mérite de permettre d'entendre en français les deux connotations qui s'y trouvent en allemand : l'événement d'une advenue et la convenance d'une appropriation (concordance, correspondance, concomitance). Nous le faisons donc nôtre ici, sans entrer dans un débat de spécialistes qui n'est pas notre objet ${ }^{3}$. Ce que Heidegger veut montrer avec son concept d'avenance (Ereignis), c'est qu'entre le sein (l'être au sens métaphysique de super-étant) et le Seyn (l'être qui se manifeste dans l'avenance et qui n'a plus rien de substantiel mais qui ouvre à la possibilité elle-même d'être), il faudra choisir. Or la langue de l'avenance, die zeigende Sage (la parole poétique révélante), seule parole appropriée conjointement à l'être et à l'homme, et de ce fait appropriante, sourd du silence effrayant et imposant du sacré originaire qui l'engendre et la meut. C'est que le parler du poète montre (zeigen). Que montre-t-il ? Un monde éthéré, lointain, désincarné? Un divin au-delà, inaccessible? Que non pas. Le Quadriparti qui émerge de la parole poétique est la jointure au contraire entre terre et ciel, mortels et divins. «L'être de la poésie [...] ne survole pas la terre, elle ne la dépasse pas pour la quitter et planer au-dessus d'elle. C'est la poésie qui tout d'abord conduit l'homme sur terre, à la terre, et qui le conduit ainsi dans l'habitation $»^{4}$. Apatride dans le lieu qu'il est lui-même, le Dasein de Sein und Zeit (1927) doit, en effet, se rendre le monde familier, usuel, et habiter ainsi la terre comme sa patrie ou son terroir (Heimat) ce mot comprend les notions de terre, de culture, de région, de contrée... Le terroir contredit l'étrangéité. Il fait du monde un lieu de possible "mienneté » (Jemeinigkeit) qui devient ainsi milieu historial, histoire d'un peuple, culture d'une langue. Le présent ainsi habité peut revêtir la paix malgré l'être-vers-la-mort, et le souci «cesse d'être 
tragique » grâce au «sceau de l'habiter " 5 . Désormais l'homme "habite le monde en poète ", selon l'expression de Hölderlin, reprise et commentée maintes fois par le philosophe allemand 6 .

4 Heidegger pense ainsi une transcendance à même l'immanence, et son sacré est désacralisé en ce sens. Les divins ou les dieux qu'il mentionne indifféremment ne sont pas plus hors-monde qu'ils n'y sont incarnés. Ils expriment plutôt cette dimension sacrée mais immanente de l'existence au monde du poète qui, précisément, n'a pas fait le pas du liturge vers la Transcendance absolue. Mais l'immanence n'est pas non plus comprise comme le seul niveau de l'étant. Il n'y a dans le sacré heideggérien ni véritable au-delà ni réel en deçà. Et là est à la fois la difficulté et l'originalité de sa pensée. Heidegger en est conscient lorsqu'il écrit: "même si l'on conçoit la "transcendance" autrement que jusqu'ici, si on la conçoit comme passage au-delà et non comme le supra-sensible à titre de véritablement étant, même avec cette détermination-là, on défigure de manière trop facile la pleine essence d'être le là. Car ainsi également, transcendance implique la présupposition d'un en-bas ici-bas ${ }^{7}$, ce qu'il récuse. Le passage en question n'est pas celui d'un monde à un autre, mais d'un temps à un autre : de la temporalité historiale tournée vers le passé, qui a fait son temps, si l'on peut dire premier commencement grec et histoire métaphysique de l'être -, à celle de l'avenance ouverte à l'à-venir où le temps a lieu d'être, c'est le cas de le dire (l'autre commencement). D'où l'écueil qu'il dénonce: celui de l'idolâtrie à même la métaphysique, qu'il nomme ailleurs l'ontothéologie, et qu'il décrit comme suit :

Chercher les Dieux au-dedans de l'étant. Si vous cherchez et que vous ne trouviez pas, par suite vous vous enferriez dans les contrefaçons de la fabrication, il n'y a plus aucune marge de liberté pour attendre dans la retenue, pour pouvoir attendre une rencontre ou même un signe qui vous soit adressés.

5 Ce qui fait défaut ici, dans la métaphysique, c'est l'absence même de défaut. Il manque le manque, la possibilité autre qui est précisément le propre de l'avenance. On cherche alors trop minutieusement ce qu'on attend: Dieu, le dieu, les dieux, du divin, peu importe. On trouve ce qu'on cherche, mais on ne se trouve pas dans ce qu'on ne cherchait pas et qui est venu nous chercher. L'avenance est cette recherche d'être cherché, ce que Heidegger nomme l'« aller à l'abîme ", au manque, ou « la non-quiétude du questionnement $"^{9}$ jamais assouvi, qui n'est pas la recherche du vide ou du néant, mais de l'Être comme l'inétant, c'est-à-dire de la possibilité de la possibilité, toujours plus haute pour lui que l'effectivitéi ${ }^{10}$. À l'extrême donc, la parole ne montre plus rien mais décèle la possibilité de la monstration elle-même. "L'Ereignis n'est autre que LE phénomène pur et simple. Il est l'inapparent toujours en retrait qui libère la possibilité

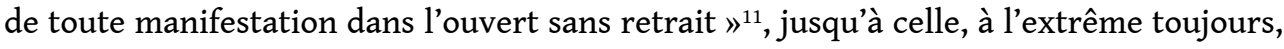
d'un dieu qui pourrait, peut-être, ou peut-être pas, passer par (ce) là.

6 La langue poétique ainsi comprise est un appel vers l'Être, une habitation auprès de l'origine, qui maintient et abrite l'ouverture à la possibilité d'une advenue à la fin, d'un dévoilement qui pourtant garde sa part de secret (le retrait de l'alètheia) ${ }^{12}$. De la sorte, elle est la modalité même de l'avenance, puisque «l'esprit de la poésie séjourne dans la proximité de la source $»^{13}$. Demeurer auprès de la source, c'est se rendre disponible à l'avenance. Personne ne sait, toutefois, pas même Heidegger, ce qui surgit véritablement dans l'autre commencement. L'homme n'existe plus vraiment au-monde, $\mathrm{ni}$ même sur la terre : il habite dans l'entre-deux du sacré, entre le présent d'ici et l'à-venir du là. 
Le poète montre cet Ouvert de l'Entre-Deux où il doit lui-même tout d'abord habiter, et il le montre de la façon que son dire, en montrant, suit l'origine, et ainsi demeure, en s'établissant fermement dans le Sacré qui doit venir dans sa parole. [...] La fondation est alors chez soi dans son être. L'habitation comme fondation est l'habitation originelle des fils de la terre qui sont en même temps les enfants du ciel. Ce sont les poètes. Leur œuvre poétique n'est tout d'abord que de fonder, c'està-dire de tracer et de jeter fermement les fondations sur lesquelles il faudra construire la maison où les dieux doivent venir en hôtes. Les poètes consacrent le $\mathrm{sol}^{14}$.

7 Plusieurs questions se posent dès lors : comment se fait-il que du sacré au divin et de la terre au ciel, nulle mention de religion ne vienne déterminer le Dasein habitant la terre ? Ce dieu qui risque de passer à l'extrême du commencement autre, et qui n'est pas Dieu mais Être (Seyn) et porche de l'Ouvert («Aître», selon la traduction de F. Fédier), qu'a-t-il de divin à proprement parler? Enfin, puisque, toujours selon Heidegger, «l'homme en tant qu'homme s'est toujours déjà rapporté à quelque chose de céleste et mesuré avec lui » ${ }^{15}$, la dimension liturgique n'aurait-elle pas à s'inviter ici, puisqu'elle est toujours pensée en termes d'entre-deux ${ }^{16}$ ?

8 Ne nous méprenons pas. Le sacré dont il est question avec le Quadriparti n'a rien de religieux au sens habituel du terme. Le « Dieu à l'extrême » qui passe furtivement dans la clairière de l'Être n'est certainement pas celui des religions. Au contraire, il est perçu chez Heidegger, après Hölderlin, comme l'expérience de la Gottlosigkeit : l'absence des dieux, leur perte, le déliement d'avec eux ${ }^{17}$. D'où la privation et le deuil, la plainte et la lamentation bien plus que la présence et l'invocation qui caractérisent le terroir de l'avenance. Aussi le sacré est-il un pur « désintéressement accompli » selon Heidegger, qui s'en explique ainsi ${ }^{18}$ :

Que voudrait d'autre le cœur endeuillé sinon, en abandonnant les dieux, sauvegarder intacte leur divinité [...], se maintenir à proximité de leur divinité. [...] La volonté de se résigner au renoncement, qu'est-ce d'autre - ce n'est rien d'autre que l'unique possibilité de rester résolument disponible à l'attente du divin. [...] Lorsque le plus aimé s'en est allé, il reste encore l'amour, car sinon le plus aimé n'aurait pu s'en aller. Le fait que les dieux se soient enfuis ne veut pas dire que le divin (die Gottlichkeit) ait disparu du Dasein de l'homme ; cela veut dire qu'il règne justement, mais sous une forme inaccomplie, une forme crépusculaire et sombre mais cependant puissante ${ }^{19}$.

9 Cette forme, qui est la dernière, ramène aussi à l'originaire, bien qu'il y ait une différence assumée entre la lumière stellaire de l'avenance et celle solaire du commencement grec. L'excès de l'absence confine l'être au néant de la présence en tant qu'il la déborde en un abîme sans fond. Il y a un " athéisme » dans l'avenance qui est le contraire d'une négation ou d'un irrespect de Dieu chez Heidegger, et qui pourrait bien porter quelques oripeaux de la religion mine de rien. Car il est pensé comme sauvegarde de la vérité du Divin : celui qui force Dieu (le dieu) à habiter le retrait, c'està-dire à garder la distance qui le sauverait de notre idolâtrie métaphysique. Nulle Révélation ici, encore moins d'Incarnation, nulle Rédemption même, et le "lien » semble distendu entre transcendance et immanence. S'il y a Salut, ce sera plutôt celui du dieu par l'homme qui seul, en étant le là de l'Être, peut faire advenir l'ajointement appropriant de l'avenance. Il est toutefois évident que le probable dieu caché au sein de l'avenance, choisissant le retrait dans sa manifestation même, et dont on ne peut approcher le secret (l'Énigme de l'Être) que par la poésie d'une parole chantée, interroge la dimension liturgique de l'homme comme telle. Or la liturgie, entendue au sens large comme l'exposition de l'orant face à l'Absolu (coram Deo) oblige à passer du sacré au 
saint ; ou, dit, autrement, de l'absence énigmatique du sacré vide de divin à la présence mystérieuse du saint plein de Dieu. En effet, s'il faut donc dépasser la métaphysique pour vivre le saut (et le sursaut) de l'avenance afin d'accéder à l'autre commencement de la pensée, à son vrai commencement ou à sa vérité (alètheia), pourquoi donc le sacré ne vivrait-il pas lui aussi un saut qualitatif ? C'est la question que nous posons à Heidegger avec J.-Y. Lacoste. Car le saut dans la clairière de l'Être que permet la dimension du sacré par le biais de la parole poétique ne pourrait-il pas (ne devrait-il pas) s'accompagner du seuil de la lisière du Royaume ${ }^{20}$ qu'offre la vie liturgique ? La parole de louange a peutêtre plus encore à dire et à montrer que la parole poétique, et le vers du poème n'est peut-être qu'un verset déloigné. Quoiqu'il en soit, Levinas a souligné «la dégénéréscence du sacré où le sacré se tient. Le sacré qui dégénère est pire que le sacré qui disparaît. C'est pourquoi le sacré n'est pas sacré, le sacré n'est pas la sainteté ", fustigeant ainsi l'incapacité de la pensée heideggérienne à passer du sacré au saint ${ }^{21}$. J.Y. Lacoste, dans cette lignée - inavouée mais bien réelle - n'a cessé lui aussi de montrer le caractère subversif du phénomène de la liturgie pour le monde du Quadriparti.

\section{La sainte lisière de Jean-Yves Lacoste}

«Nul n'entre en liturgie sans vouloir que Dieu le visite $»^{1}$. La logique de la liturgie est une présentation à Dieu, une exposition à son Absolu, à son advenue. Elle est celle de la possibilité d'entrer en relation avec Celui qui échappe à notre finitude, mais dont la transcendance elle-même ne le rend pas pour autant inaccessible (sinon pourquoi s'y exposer?). Un paradoxe qui oblige l'existence à avouer qu'elle ne se suffit pas à ellemême, mais qu'elle ne trouve son sens que dans un horizon eschatologiquement relationnel. J.-Y. Lacoste souligne que l'être-au-monde est avant tout coram Deo ${ }^{2}$. Heidegger lui-même a utilisé la formule, dans son cours de 1920-1921 sur la Première épître de Saint Paul aux Thessaloniciens, comme une catégorie de la facticité chrétienne, mais en la tronquant intentionnellement de toute référence liturgique ${ }^{3}$. Pour lui en effet, le rapport de l'homme à Dieu est de l'ordre existentiel-ontique et non existential-ontologique, ce qui signifie qu'il touche à la superficie de son être et non au fondamental de l'humain 4 . Mais pour Lacoste, l'esse coram Deo n'est pas un mode d'être superfétatoire. Il ne surplombe pas l'être facticiel. Il le subvertit en rendant la facticité secondaire, face à l'entrée de l'eschaton dans son champ. Là, Lacoste rejoint la dimension hespérique de l'avenance heideggérienne. Mais il la pense dans une rencontre de personne à personne, ce qui bouleverse la donne. Un passage du Monde et l'absence d'œuvre nous paraît résumer à l'envi ce qui vient d'être dit :

[La liturgie] échappe au rythme oscillant qui unit "profanité" et "sacralité", monde désenchanté et terre numineuse. Elle est œuvre libre de rupture. La liturgie laisse le monde derrière elle parce qu'elle subordonne la relation d'inhérence à une relation d'affrontement (coram) [...], elle laisse la terre derrière elle parce que [elle ne peut] se satisfaire de l'eschatologie de substitution que lui offre la protection présente $\mathrm{du}$ sacré. La liturgie n'a pas lieu ailleurs que dans le monde et sur la terre [...] mais, dans le monde ou sur la terre, elle ouvre un nouvel espace et un nouveau rapport au réel : symboliquement l'homme liturge anticipe ici ou là ce que la théologie pense sous le concept de Royaume ${ }^{5}$.

11 Aussi J.-Y. Lacoste dénonce-t-il fermement dans Le monde et l'absence d'œuvre la " phénoménologie de l'expérience païenne » de Heidegger ${ }^{6}$. Avec la notion d'Ereignis, ou de l'Être comme avènement du Quadriparti dissimulé dans l'événement de la parole 
poétique, Heidegger est certes sorti du monde profane du seul être-vers-la-mort, mais ne franchissant pas le seuil du saint, il aurait reculé à force de pas en arrière dans un Sacré douteux. Le silence du retrait secret au sein de l'avenance a, en effet, toutes les caractéristiques du numineux sous couvert de lumineux. Ce sacré-là, en vérité, «n'a rien à dire. Il se saisit de l'homme dans les phénomènes de la fascination et de l'effroi une expérience plus brutale que celle de l'infini $»^{7}$, note Lacoste, qui ajoute : le sacré " apparaît comme quelque "chose" et non pas comme "quelqu'un" », or « seul parle le "quelqu'un"; le "quelque chose" ne parle pas $»^{8}$. Quelle langue peut bien parler l'avenance s'il n'y a pas en elle de rencontre ? Une parole non adressée, toute poétique soit-elle, un questionnement renouvelé non échangé, tout philosophique soit-il, sont lettre morte. L'altérité qui se joue dans l'avenance (l'autre commencement) reste sans présence. La parole poétique ne donne lieu à aucun dialogue, à aucun échange. Ce qui résonne en elle n'est que son propre écho. Son appel s'abîme dans le néant, son silence glacé ne fait pas éclore de répons(e). Car si l'être approprie à soi, il ne relie à rien d'autre que soi. Il n'est pas personnel donc il n'est pas relationnel. Il manque au sacré la promesse d'une alliance. Il lui manque précisément ce qui fait la «religion », le fait de relier, de faire du lien, et de pouvoir relire une histoire sainte en commun. Être approprié à soi, à l'être lui-même, sans être relié à un autre, qu'est-ce que cela donne ? Rien. On n'est pas seulement soi-même comme un autre (Ricoeur), on est aussi et avant tout soimême pour un autre (Levinas). Et Lacoste de renchérir :

En Dieu seul l'être est, simplement, relation; et l'homme avoue sa finitude de créature à n'être pas que relation, à exister toujours substantiellement dans l'identité du soi à soi-même, avant de se déporter vers l'autre et vers Dieu dans l'être relationnel. Il demeure que c'est là précisément que l'homme est plus humain, dans ce déport ou cet abandon qui est [...] le plus certain de sa définition'.

12 Aussi peut-il s'ennuyer du sacré heideggérien, se divertir d'une avenance dans laquelle au final rien n'advient ${ }^{10}$, et rêver d'un au-delà du monde factice et de la terre sacrale. Le profane ou le sacré ne lui suffisent pas. Il est fait tout entier pour la relation, et la relation absolue, à l'Absolu, ce que Levinas nomme sans ambages la sainteté. Le Souci du Dasein ne saurait empêcher l'inquiétude, au sens augustinien du terme, de le tarauder; inquiétude qui lui donne de se découvrir comme liturge : «Tu nous as créés pour toi, Seigneur, et notre cœur est inquiet [sans repos, sans lieu, sans là] tant qu'il ne demeure en Toi $»^{11}$. La poésie dans l'avenance heideggérienne donnait l'habitation d'une manière quasi sacrale, mais la louange liturgique offre linhabitation de la modalité sacramentelle, la demeurance en commun et mutuelle. En elle seule se noue le véritable appropriement non plus de l'être et de l'être-là, mais de Dieu et de son Ecclesia, ou assemblée des coram Deo. "Dieu peut nous apparaître [alors] sous les traits de l'inesquivable, mais ce sera le cas dans un autre monde, celui de la foi et des attitudes ou jeux de langages que je n'aime pas dire "religieux", mais plutôt "liturgiques" », précise Lacoste $^{12}$. Pourquoi une telle réticence de vocabulaire? Car l'attitude religieuse contenue dans le sacré «se contente en fait d'assez peu $»^{13}$ ne donnant pas Dieu à proprement parler, mais toujours l'idole (du Suprême de la métaphysique ou de l'extrême du divin, peu importe). Pour qu'il y ait Dieu, il faut plus que de l'existence. Il faut la sainteté de se recevoir ensemble d'une Présence célébrée. C'est bien toujours de l'habiter qu'il s'agit, mais en une modalité de l'avenance totalement nouvelle et impensée du philosophe allemand (intentionnellement, n'en doutons pas). Celle que nous nous permettons de nommer l'avenance liturgique, dans la lignée de la critique lacostienne du sacré heideggérien. Et qu'il nous faut à présent à tout le moins esquisser. 


\section{L'Autre que l'Être : vers une avenance liturgique?} Avec l'Ereignis, Heidegger a posé philosophiquement la question de l'eschaton en proposant de l'envisager sur une modalité résolument ontologique. Mais si l'homme reçoit bien son présent à partir de son avenir tout imprégné d'un passé non révolu, la question de l'avenance reste de savoir de quelle eschatologie il vit les prémisses, de quelle donation il est le témoin. N'y aurait-il pas une autre modalité, non seulement possible mais nécessaire, pour la penser jusqu'au bout - pour ne pas dire jusqu'à l'extrême? Aux abords de la clairière heidegerienne (Lichtung) se dessine comme à contre-jour la lisière lacostienne (coram Deo). C'est pourquoi la réflexion sur l'Ereignis peut éclairer a contrario ce qu'il en est de ce seuil pré-eschatologique dans lequel nous sommes pris, en liturgie.

La passée du sacré au saint, en effet, oblige l'hespérique à n'être plus l'attente solitaire de l'Être furtivement à venir, la garde consciencieuse des dieux absents ou fuyants au crépuscule, ni la remontée plus ou moins sereine auprès de sa source somme toute inatteignable. Car le commencement autre heideggérien n'est pas si originaire que cela, il initie une nouvelle donne sans offrir pour autant l'alètheia de l'origine que la liturgie nomme le Dieu Créateur ou la Présence mystérieusement hospitalière de son Verbe. Si l'origine n'est peut-être pas, pour nous, donnée au commencement - car elle nous échappe en son secret -, la liturgie nous enseigne qu'elle l'est sûrement d'une manière ou d'une autre à la fin, par ce que J.-Y. Lacoste nomme une "relance de l'expérience $~^{1}$ qui nous fait entrevoir non le retrait de l'Être, mais le Mystère de l'Autre. Notre propre naissance, certes, nous est inaccessible, mais une renaissance peut s'envisager en liturgie aux abords de l'eschaton, qui lui donne sens ainsi, comme co-naissance, naissance en un Autre, par un Autre, avec d'autres. Mystère de don et d'amour mutuels, de surprise et de vie nouvelle, alpha et omega, qui toujours s'efface en son advenue dans une manière autre que la Gottlosigkeit. L'abîme ici n'est pas absence mais mystère d'une altérité qu'on n'aura jamais fini d'explorer, et qui se nomme communion. Mystère d'une présence qui se refuse à s'imposer mais se laisse seulement deviner, à même l'assemblée. Présence discrète, secrète donc, sans ostentation, furtive aussi en ce sens qu'elle échappe à toute tentative de mainmise, mais qui toutefois ne peut se cacher totalement puisqu'elle se pressent dans la grâce - le charme - qu'elle dégage presqu'à son insu pour qui se dispose à aimer' ${ }^{2}$. Dieu est plus avenant que l'Être ! Car il est bien moins abstrait.

Il y a, en effet, un pressentiment de l'Absolu en liturgie qui n'est pas un sentir à proprement parler, mais qui possède la certitude et l'évidence qui se dégagent d'une présence, invisible cependant, invue souvent, imparable pour autant. L'orant « ne fait jamais face à un tout-connu, mais à un connu-et-inconnu. [...] Dieu se donne comme celui qui apparaît sans tromper mais en transcendant cette apparition. [...] L'inévidence, donc, n'est pas l'autre de l'évidence, mais un mode de celle-ci. [...] Inévidence ou mi-évidence...telle serait donc l'évidence qui a lieu dans l'élément du mystère $»^{3}$. Mystère, le mot revient, et sans doute dit-il le mieux de quoi il en retourne en liturgie, désignant ainsi la lisière d'un Royaume qui n'est pas de ce monde. Celle-ci pourtant a ceci de paradoxal qu'elle est un seuil qui ne peut être appréhendé de loin, du dehors. C'est de l'intérieur que l'on passe du sacré au saint. Il y faut une appropriation personnelle, pour ne pas dire un appropriement du Royaume lui-même. La présence 
divine n'est pressentie que dans une exposition de soi appropriée à elle, reconnue comme son propre. Le Mystère a cette caractéristique, Gabriel Marcel nous l'a appris, d'être ce dans quoi l'on est pris. Nous faisons partie de lui, et son intime fait partie de nous. Il n'est ni l'énigme, obscure, qui peut se résoudre par nos propres forces, ni le problème, dur, sur lequel nous buttons car il nous reste extérieur. D'où un lien intrinsèque entre mystère et relation. Car, comme le souligne encore Marcel, «toute présence est mystérieuse, et il est plus que douteux que le mot mystère puisse être employé là où une présence n'est pas au moins pressentie $»^{4}$. De là une modalité de l'avenance tout à fait autre en liturgie. Elle substitue au poétique une note relationnelle (dimension de lien et d'altérité), pascale (dimension de traversée et de renaissance), et même sacramentelle (dimension de mystère - sacramentum - et de présence), qui fait de l'avenance une nouvelle modalité d'existence à pleinement investir, et non une pure énigme de la pensée à seulement présager.

Ce nouveau langage, silencieusement ou choralement dialogal, ouvre un espace inédit jusqu'ici. En lui l'apérité à l'Être se meut en une ouverture à l'Autre qui est bien un commencement autre, mais celui d'un autrement qu'être (Levinas). Celui d'une communauté de célébration et d'espérance au seuil du saint lorsqu'elle vit au cœur du mystère. De cet autre trait caractéristique de la liturgie, la louange, il faudrait donc aussi parler puisque l'avenance se dit en chantant ${ }^{5}$. Mais si la poésie est chant (Lied) solitaire et monocorde, la liturgie, elle, ne saurait louer sans responsorialité et sans pluralité dans l'harmonie ${ }^{6}$. En tant qu'exposition en commun, à plusieurs, polyphonique, et délibérée, elle est disponibilité communautaire à une possibilité sans précédent d'êtres en-alliance dans l'embrasure du Royaume. Elle n'est pas l'ouverture à tous les possibles de l'être en avenance, dans la neutralité du Rien à-venir, mais la possibilité inouïe et multiforme de Dieu en présence, dans la réalité de ce que l'on pourrait appeler le Touten-surprise. Car il faut que l'attente soit bouleversée et la rencontre inespérée pour que Dieu soit Dieu, c'est-à-dire qu'il échappe à toute mesure humaine, trop humaine (Nietzsche). Le pré-eschatologique en liturgie ne sera donc peut-être pas autre chose que ce pressentiment de présence parousiaque entraperçue et espérée en commun à travers le mystère d'un don infini qui nous prend en lui. On est bien loin de l'eschatologie de l'être où le Secret tout avenant qu'il soit n'a aucun charme de présence et ne délivre en aucun cas de la facticité de l'existence. L'apérité liturgique devient très précisément, dès lors, celle de la possibilité infinie, et non pas indéfinie, d'une Présence qui, tout en nous échappant, nous définit en propre comme sa propriété - en l'occurrence son Corps, son Peuple ou son Épouse ${ }^{7}$. Se fait jour alors une possibilité autre que la finitude : celle de l'inespéré d'une Présence si absolue qu'elle déjoue jusqu'à la mort. De là à déduire que le Christ accomplit dans une certaine mesure la figure même de l'avenance, ou plutôt qu'il manifeste en sa Pâque que l'avenance ne saurait se suffire à elle-même, il n'y a qu'un pas que Jean Vioulac a franchi hardiment. Il put le faire parce que Hölderlin lui-même avait identifié le dieu à l'extrême (ou dernier dieu) et le Christ Jésus, en tant que celui-ci accomplit en sa Kénose l'abîme de la déité qui se vide d'ellemême en son advenue chez nous et pour nous. Ce que Heidegger a toujours refusé de voir, amputant le poète de la fine pointe de son inspiration, en une lecture "partiale voire de mauvaise foi $»^{8}$. Là encore, il a délibérément manqué le saint :

Tout le cheminement de Heidegger se rassemble dans la pensée d'un "autre commencement", que seul le passage d'un "dernier dieu" serait susceptible de provoquer, en instituant, par-delà l'expérience grecque elle-même, une "tout autre vérité", définie par la sauvegarde du mystère. [...] Dire que Jésus est Christ, c'est 
dire qu'il est Commencement [...]. Il est alors possible d'objecter à Heidegger que l'"autre commencement", précisément tel qu'il l'a conçu, a déjà eu lieu. [...] Cette autre histoire, celle de la sauvegarde du sacré et de la dénomination du saint, est l'histoire sainte, et depuis lors, ces deux histoires, disait saint Augustin "avancent ensemble, enchevêtrées l'une dans l'autre" 9

17 De cette histoire devenue sainte, la liturgie est le Mémorial, offrant dans l'action de grâce (eucharistia) un chant d'anamnèse qui fait étonnamment mémoire du futur à venir, de cette Présence vivante au-delà de la mort et avenante au-dedans du mystère, transfigurant ceux qui y communient. «Ce qui advient avec l'expérience chrétienne, c'est une nouvelle conception de l'eschatologie [...] : [non] pas l'attente d'un événement futur mais l'éveil à l'imminence de cette venue " $^{0}$, déjà à l'œuvre dans la liturgie (Opus Dei). Expérience originale donc, de venue en présence jamais achevée mais déjà accomplie, que l'on hésite même à qualifier de la sorte, car paradoxalement souvent insensible, inau-dible, indicible. Lacoste insiste et martèle : l'expérience liturgique est toujours l'expérience d'une inexpérience, car Dieu déborde tout cadre et toute immanence. Le retrait dans la manifestation a donc toute sa place en liturgie - et c'est ce qui fait que nous pouvons parler d'avenance liturgique sans aberration - mais d'une toute autre manière que celle de l'inapparent de l'Être heideggérien. Car l'effacement dont il s'agit, voire la désappropriation surprenante qu'il trahit et que la théologie nomme Kénose, n'est ni un refus de se dire ni une manière de se dédire, mais une Parole en elle-même plus « montrante » que toute poésie. Le Verbe ou le Logos dont la vérité est par là dévoilée (apokalupsis) ne se révèle pas seulement appropriant ou approprié, il parle en propre, en personne. Il est Quelqu'un. Donné, livré, abandonné à la disponibilité ou non d'une réponse en forme de répons. Voilà le saut, le passage, la traversée qu'opère le liturgique en obligeant les hommes en existence à quitter la clairière de l'Être pour s'exposer délibérément à la lisière d'un Autre, faisant d'eux, non plus les avenants du sacré mais les chantres du Saint

\section{NOTES}

1. Concept de M. Eliade, in Le sacré et le profane, 1956, dans la lignée de R. Otto qui avait proposé celui de "numineux » in Le Sacré dès 1917. Cf. J. Ries, L"homo religiosus" et son expérience du sacré, Paris, Cerf, 2009.

2. Odon Casel publie en 1932 Das christliche Kultmysterium (Le mystère du culte dans le christianisme, Paris, Cerf, 1964, «Lex Orandi » nº). Louis Bouyer y réagit en 1945 dans Le mystère pascal (Paris, Cerf, 1957, « Lex Orandi » $n^{\circ}$ 4, p. 17 sq).

3. L. Bouyer, Le rite et l'homme (1962), Paris, Cerf, 2009, p. 115-116.

4. Que l'on songe par exemple à la parabole du fils prodigue qui à elle seule inspire dans les années 1910 à la fois Gide, Lucien Jean, Rilke, Péguy et même Kafka. Nous renvoyons pour plus de détails à notre article « La parabole vagabonde ", Sources vives, $\mathrm{n}^{\circ}$ 187, sept. 2017, p. 28-35.

1. Religare, relier et relegere, relire, sont les deux étymologies possibles du mot religion. On trouve le sens de relegere chez Cicéron (La nature des dieux, Livre II, XXVIII, § 72, Paris, Les Belles Lettres, 2002, p. 89) et celui de lien chez Saint Augustin (La Cité de Dieu, Livre X, § 3, Paris, Institut d'Études augustiniennes, 1993, « Nouvelle bibliothèque augustinienne » 3, p. 550). 
2. M. Heidegger, Essais et Conférences, trad. par A. Préau, Paris, Gallimard, 1958, p. 234.

3. Nous savons la controverse autour de la traduction de F. Fédier et ne faisons allégeance ni à Heidegger ni à ses traducteurs quels qu'ils soient en utilisant sciemment le mot «avenance » ici. Il nous a semblé simplement plus judicieux dans le cadre d'une philosophie de la liturgie d'éviter le mot «événement » qui offrirait d'emblée une connotation pascale et théologique qu'Ereignis n'a pas ni ne veut avoir. Le terme « avenance » est tellement inédit qu'il évite toute confusion, et nous paraît plus heureux à tout prendre qu' " amêmement » (autre essai de traduction d'Ereignis).

4. M. Heidegger, Essais et conférences, op. cit., p. 230.

5. J.-Y. Lacoste, Expérience et Absolu, Paris, PUF, 1994, p. 17.

6. "L'homme habite en poète " (conférence du 6 octobre 1951). On le sait, Heidegger consacre des cours entiers et de nombreux discours à Hölderlin (GA 4 et 75, trad. par H. Corbin, M. Deguy, F. Fédier et J. Launay in Approche de Hölderlin, Paris, Gallimard, 1973, et Hymnes de Hölderlin, Paris, Gallimard, 1988). Cf. aussi E. Brito, Heidegger et l'hymne du sacré, Louvain, Peeters, 1999.

7. M. Heidegger, Apports à la philosophie, De l'avenance, trad. par F. Fédier, Paris, Gallimard, 2013, p. 369 (GA t. 65, p. 322).

8. Ibid., p. 458 (400).

9. Ibid., p. 454 (397).

10. «Plus haut que l'effectivité se tient la possibilité » (Heidegger, Être et Temps, fin du § 7).

11. H. France-Lanord, « Ereignis » in Dictionnaire Martin Heidegger, P. Arjakovsky et al., Paris, Cerf, 2013, p. 406.

12. Cf. D. Franck, Le nom et la chose, Paris, Vrin, 2017, p. 29 : «L'homme est à l'écoute de l'appel de l'être et l'appartenance de l'un à l'autre caractérise la langue [...] où réside notre essence. N'est-ce pas dire que l'être lui-même - ou l'Ereignis qui en est le nom propre - est co-appartenance de l'appel et de l'écoute : langue?".

13. M. Heidegger, Approche de Hölderlin, op. cit., p. 117.

14. Ibid., p. 190.

15. M. Heidegger, Essais et conférences, op. cit., p. 234.

16. Ainsi le «déjà-pas-encore " d'Oscar Cullmann in Christ et le temps, Temps et Histoire dans le christianisme primitif, Neuchâtel-Paris, Delachaux et Niestlé, 1947.

17. «Hölderlin est bien, par excellence le poète du sacré. Non certes parce que ses poésies contiennent les noms des dieux mais parce qu'il fait l'expérience de l'absence de Dieu» (B. Allemann, Hölderlin et Heidegger, Paris, PUF, 1987, p. 159).

18. M. Heidegger, Hymnes de Hölderlin, op. cit., p. 89.

19. M. Heidegger, ibid., p. 96.

20. Nous devons le terme lisière à Jérôme de Gramont, Lacoste utilisant plutôt ceux de "marge » ou « limite ».

21. E. Levinas, Du sacré au saint, cinq lectures talmudiques, Paris, Les Éditions de Minuit, 1977, p. 109.

1. J.-Y. Lacoste, Expérience et Absolu, op. cit., p. 76.

2. L'expression coram Deo (« se tenir devant Dieu », « devant sa Face ») est avant tout biblique, mais on la trouve conceptualisée comme telle dès St Augustin, reprise par Luther, Kierkegaard, Levinas, Henry... Lacoste dit l'avoir reçue avant tout de Ebeling dans sa Dogmatik der christlichen Glaubens (Tübingen, 1979, I § 64, cité in Présence et Parousie, Genève, Ad Solem, 2006, p. 68). L'originalité de Lacoste n'est donc pas tant dans ce qualificatif de l'humain que dans la place centrale qu'il lui attribue dans sa réflexion.

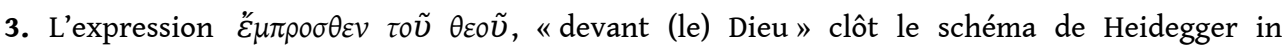
Phénoménologie de la vie religieuse, Paris, Gallimard, 2012 (Ga 60, p. 96). Jean Greisch commente ce fait ainsi : "La question cruciale est celle des raisons qui ont empêché Heidegger d'inclure la célébration liturgique dans sa description du coram Deo. S'agit-il d'un silence purement 
accidentel, ou dénature-t-il l'analyse heideggérienne du christianisme ? La réponse de J.-Y. Lacoste ne fait pas l'ombre d'un doute " (L'arbre de vie et l'arbre du savoir, Paris, Cerf, 2000, p. 215).

4. Cf. J.-Y. Lacoste, Être en danger, Paris, Cerf, 2011, p. 141 note 1.

5. J.-Y. Lacoste, Le monde et l'absence d'œuvre, et autres études, Paris, PUF, 2000, p. 16-17.

6. Ibid., p. 11.

7. J.-Y. Lacoste, Recherches sur la parole, Louvain-la-Neuve, Peeters, 2015, p. 168.

8. Ibid., p. 174

9. J.-Y. Lacoste, « Du désir de Dieu, méditation théologique », Résurrection, n 69, 1982, p. 114-115.

10. Heidegger le dit lui-même : «Im Ereignis geschieht nichts » (Dans l'Ereignis, rien n'advient), GA 97, p. 382, d'où l'on comprend qu'il soit difficile de traduire Ereignis par événement.

11. Saint Augustin, Les Confessions, Paris, Garnier-Flammarion, 1964, L. I, chap. 1, p. [15]. Trad. modifiée.

12. J.-Y. Lacoste, Présence et parousie, op. cit., p. 228.

13. Ibid., p. 225.

1. J.-Y. Lacoste, Note sur le temps, Paris, PUF, 1990, p. 201.

2. " "Aimer" s'entend ici comme disposition plus (souvent) que comme occurrence. [... ] Les choses nous apparaissent en s'imposant à nous. [...] L'amour toutefois, lorsqu'il fait acte d'apparition, contredirait son essence ou son propos s'il faisait œuvre de contrainte» (J.-Y. Lacoste, La phénoménalité de Dieu, Paris, Cerf, 2008, p. 94-95).

3. J.-Y. Lacoste, «Quand je parle de Dieu... » in Dieu en tant que Dieu, la question philosophique, P. Capelle-Dumont (éd.), Paris, Cerf, 2012, p. 219-220.

4. G. Marcel, Le mystère de l'être, Livre I, Paris, Présence de G. Marcel, 1997, p. 232.

5. Cf. M. Heidegger, Acheminement vers la parole, Paris, Gallimard, 1976, p. 255.

6. D'où il appert que le prisme lévinassien du face-à-face relationnel n'est sans doute pas suffisant pour dire ce qui se joue en liturgie dans le coram Deo. Ce qu'il faudrait creuser, mais là n'est pas notre propos.

7. Images bibliques traditionnelles pour désigner l'Église en Christianisme, reprises par le Concile Vatican II qui, toutes trois, insistent à leur manière sur une appropriation mutuelle, donc un appropriement en langage heideggérien : son corps dont il est indissociablement la tête, le peuple de Dieu élu irrévocablement, l'épouse sienne qu'il s'est unie indissolublement.

8. J. Vioulac, Apocalypse de la vérité, Paris, Ad Solem, 2014, p. 243.

9. Ibid., p. 226-227. La citation d'Augustin est tirée de La cité de Dieu, I, 35.

0. F. Dastur, Heidegger et la pensée à venir, Paris, Vrin, 2011, p.229. 